\title{
Subject Preferences and Psychological Implications of Portable Oxygen Concentrator Versus Compressed Oxygen Cylinder in Chronic Lung Disease
}

\author{
Pasquale Moretta, Antonio Molino, Michele Martucci, Salvatore Fuschillo, Alberto De Felice, \\ Pietro Guida, Andrea Motta, Michele Vitacca, and Mauro Maniscalco
}

\begin{abstract}
BACKGROUND: Oxygen therapy represents the elective therapy to improve the quality of life for patients with chronic respiratory diseases like COPD and interstitial lung disease. Lightweight portable oxygen concentrators (POCs) are a valid alternative to traditional systems such as portable compressed oxygen cylinders. However, patient preference and the possible psychological implications related to the use of both devices have been poorly assessed. We sought to evaluate patient preference between the ambulatory oxygen systems (ie, a POC or a small cylinder) for patients with COPD and interstitial lung disease experiencing exertional desaturation in a rehabilitation setting. Furthermore, the use of one device in comparison with the other was related to specific mechanical characteristics and related to perceived quality of life, anxiety, and depressive symptoms. METHODS: 30 subjects with COPD and interstitial lung disease, who demonstrated exertional desaturation on room air during 6-min walk test (6MWT), were recruited. Each subject performed 2 6MWTs, in random order: one breathing oxygen via a POC and one with a portable compressed oxygen cylinder. Both devices were set up to ensure oxyhemoglobin saturation between $92 \%$ and $95 \%$ during the 6MWTs. All subjects completed a questionnaire assessing anxiety, depression, and quality of life. Each device was randomly assigned to each subject for 1 week, and then replaced with the other in the following week. At the end of the trial period, all subjects completed a questionnaire evaluating several aspects of the oxygen therapy devices. RESULTS: There were no significant differences in oxygen saturation or the mean distances achieved during the 6MWTs between the 2 portable oxygen devices. The subjects expressed greater preference for the POC (73.3\%), basing their choice mainly on ease of transport and lower weight. Subjects' age also correlated with preferences: younger subjects were more negatively focused on the weight of the portable compressed oxygen cylinder, whereas older subjects considered the POC easier to manage. No significant differences in preferences were present between COPD and interstitial lung disease. CONCLUSIONS: The POC and the portable compressed oxygen cylinder performed in a comparable manner during 6MWT for subjects with COPD and interstitial lung disease and exertional desaturation. Subjects preferred the POC because it was associated with better mobility. Key words: ambulatory oxygen; long-term oxygen therapy; portable oxygen concentrator; rehabilitation. [Respir Care 2021;66(1):33-40. (C) 2021 Daedalus Enterprises]
\end{abstract}

\section{Introduction}

Many patients with severe chronic respiratory diseases such as COPD and interstitial lung disease develop

Dr Moretta is affiliated with the Neurology Rehabilitation Unit, ICS Maugeri SpA SB, Institute of Telese Terme, Benevento, Italy. Dr Molino is affiliated with the Respiratory Division, Department of Respiratory Medicine, Federico II University, Naples, Italy. Drs Martucci, Fuschillo, hypoxemia during exercise or sleep, and, as the disease progresses, this can also occur at rest. ${ }^{1}$ Exercise-induced oxyhemoglobin desaturation favors the exercise breathlessness and exercise limitation often observed in patients with both

De Felice, Guida, and Maniscalco are affiliated with the Pulmonary Rehabilitation Unit, ICS Maugeri SpA SB, Institute of Telese Terme, Benevento, Italy. Dr Motta is affiliated with the Institute of Biomolecular Chemistry, National Research Council, Naples, Italy. Dr Vitacca is 
COPD and interstitial lung disease, therefore reducing autonomy and life satisfaction and negatively affecting their quality of life (QOL). ${ }^{2}$ Domiciliary long-term oxygen therapy (LTOT) is an important option for patients with chronic hypoxemia and COPD. It seems to reduce exertional dyspnea and improves exercise performance, oxygen saturation, and survival, although conflicting results have also been reported..$^{3-5}$

Although it is unclear what mechanisms underlie the survival benefit of LTOT (eg, possibly improved oxygenation with reduced hypoxemia), patients are positively affected when the supplemental oxygen achieves a $\mathrm{P}_{\mathrm{aO}}>60 \mathrm{~mm}$ $\mathrm{Hg}$ for $\geq 15 \mathrm{~h} / \mathrm{d} .{ }^{6}$ As a consequence, the issue of LTOT prescription adherence is of utmost importance. In general, patients' adherence to LTOT prescription is suboptimal and influenced by a number of factors such as disease severity, acceptance of treatment, patient education, and availability of suitable ambulatory devices. ${ }^{7}$ Patients are often reluctant to adhere to LTOT treatment, and significant depressive symptoms or depression associated with disease are highly prevalent in these patients. ${ }^{7}$ In addition, LTOT has been associated with increased feelings of anxiety and shame, potentially leading to social isolation and restrictions in activities in daily life. ${ }^{8}$

Various devices (eg, gaseous oxygen, liquid oxygen, and oxygen concentrators), each with advantages and disadvantages, are available to deliver LTOT, generally via a nasal cannula. ${ }^{9}$ Liquid oxygen combines a large stationary unit with a small portable compressed oxygen cylinder (referred to as a small cylinder), allowing the patient to leave home while using supplementary oxygen. Portable liquid oxygen and gaseous oxygen-delivery devices have drawbacks, mainly related to their dependence on a delivery service. Compared to gaseous oxygen, patients prefer liquid oxygen because it lasts longer, filling the canister is simpler, and the portable system is easier to carry due to a lighter weight.

Recently, various portable oxygen concentrators (POCs) have emerged as an alternative to liquid oxygen devices. ${ }^{10}$ POCs draw air through a zeolite material to absorb nitrogen and provide $95 \% \pm 3 \%$ oxygen to the patient, and, unlike liquid oxygen devices, they do not need to be refilled. ${ }^{10}$ As a consequence, oxygen therapy can be performed where and when patients want, the only constraint being the

affiliated with the Pulmonary Rehabilitation Unit, ICS Maugeri SpA SB, Institute of Lumezzane, Brescia, Italy.

The authors have disclosed no conflicts of interest.

Correspondence: Mauro Maniscalco MD PhD, Istituti Clinici Scientifici Maugeri IRCCS, Via Maugeri 4, 27100, Pavia, Italy. E-mail:

mauro.maniscalco@icsmaugeri.it.

DOI: $10.4187 /$ respcare.07829

\section{QUICK LOOK}

\section{Current knowledge}

Several studies have compared lightweight portable oxygen concentrators (POCs) with traditional portable systems such as compressed oxygen cylinders. However, patient preference and the possible psychological implications related to the use of both devices have not been assessed.

\section{What this paper contributes to our knowledge}

In subjects with chronic respiratory failure and exercise desaturation, performance of portable oxygen concentrators and compressed oxygen cylinders was comparable during the 6-min walk test. The portable oxygen concentrator was preferred over the compressed oxygen cylinder due to the greater mobility allowed by this device.

availability of an electricity source to recharge the battery with a frequency depending on the flow setting, breathing frequency, and battery age. These advantages can positively impact patient adherence and QOL.

The literature data on direct comparisons between POC and liquid oxygen devices is limited, and current guidelines on domiciliary oxygen therapy do not give indications on the choice of the delivery device. ${ }^{11}$ Furthermore, patient preference for available devices and the impact on QOL have not been carefully assessed..$^{10,12}$

The aim of this study was to evaluate subject preference between 2 ambulatory oxygen systems (POC vs small cylinder) in subjects with chronic COPD and interstitial lung disease presenting with exertional desaturation, and to evaluate whether the use of one device or the other was related to differences in perceived QOL and other psychological measures.

\section{Methods}

\section{Study Design}

A randomized crossover study was conducted at the rehabilitative pneumology unit of ICS Maugeri of Telese Terme (Benevento, Italy). The study population was recruited in the Department of Respiratory Rehabilitation. Patients with COPD and interstitial lung disease with or without resting hypoxemia, but with exertional desaturation (defined as change in mean arterial saturation $\geq 4 \%$ or nadir of $88 \%$ on room air during the $6 \mathrm{MWT}$ ) who were admitted into the pulmonary rehabilitation program were eligible for this study. To be included in the study, subjects had to be clinically stable in the previous 4 weeks, 


\section{Portable Concentrator vs Oxygen Cylinder}

without comorbidities that interfered with ambulation. None of the subjects were using any type of oxygen therapy before enrollment. The study was approved by the local hospital ethics committee.

We selected a locally available POC with good oxygendelivery capacity and portability features (SimplyGo Mini, Respironics, Murrysville, Pennsylvania) and the following technical characteristics: weight, $2.3 \mathrm{~kg}$; height, $23.9 \mathrm{~cm}$; noise level, $43 \mathrm{~dB}$. The compressed oxygen cylinders used were CH-type with a weight of $4.2 \mathrm{~kg}$, a volume of $470 \mathrm{~L}$, and a height of $52 \mathrm{~cm}$. Additional weight was added to the POC to match the weight of the oxygen cylinder.

Eligible subjects performed 3 6MWTs over 2 consecutive days. The tests were conducted by senior clinicians according to current guidelines (Fig. 1). On Day 1, a 6MWT was performed while breathing room air to determine subjects' eligibility. Subjects performed a second session on the following day. On Day 2, subjects had an arterial blood gas taken on room air after a 30-min rest. Then subjects undertook 2 6MWTs, one with a compressed oxygen cylinder and the other with the SimplyGo Mini POC carried on the shoulder, in random order as determined using sealed envelopes. Between tests, subjects rested for at least $30 \mathrm{~min}$ breathing room air prior to switching to the alternate device for the second test. The lowest $\mathrm{S}_{\mathrm{pO}_{2}}$ value, maximum heart rate, and the distance walked were recorded during the 6MWT. Dyspnea and fatigue were measured using the 10-point Borg scale before the start and at the end of each test. After the 6MWTs were completed, the compressed oxygen cylinder and the SimplyGo Mini device were assigned in random order to the subjects for one week and then alternated for the second week. At the end of the trial period, all subjects completed a questionnaire evaluating several aspects of oxygen therapy devices. Psychological measures of QOL, anxiety, and depression were assessed on Day 1, while subjects' preferences were measured at the end of the study.

\section{6-Min Walk Test}

The 6MWT was performed according to American Thoracic Society recommendations. ${ }^{13}$ The test was performed in a $15-\mathrm{m}$, air-conditioned hospital corridor. Subjects were instructed to walk as far as possible in 6 min. The walk was limited by symptoms, so subjects were allowed to stop, if necessary, but they were asked to continue again after resting.

Oxygen saturation $\left(\mathrm{S}_{\mathrm{pO}_{2}}\right)$ and heart rate were monitored throughout the test via a finger electrode (SIMS-BCI 3303, Waukesha, Wisconsin). Before and at the end of the test, $\mathrm{S}_{\mathrm{pO}_{2}}$, heart rate, and blood pressure values were recorded. The minimum $\mathrm{S}_{\mathrm{pO}_{2}}$ value recorded at the end of 6MWT was considered in the analysis. Subjects were asked to rate their dyspnea and fatigue both at the beginning and at the end of

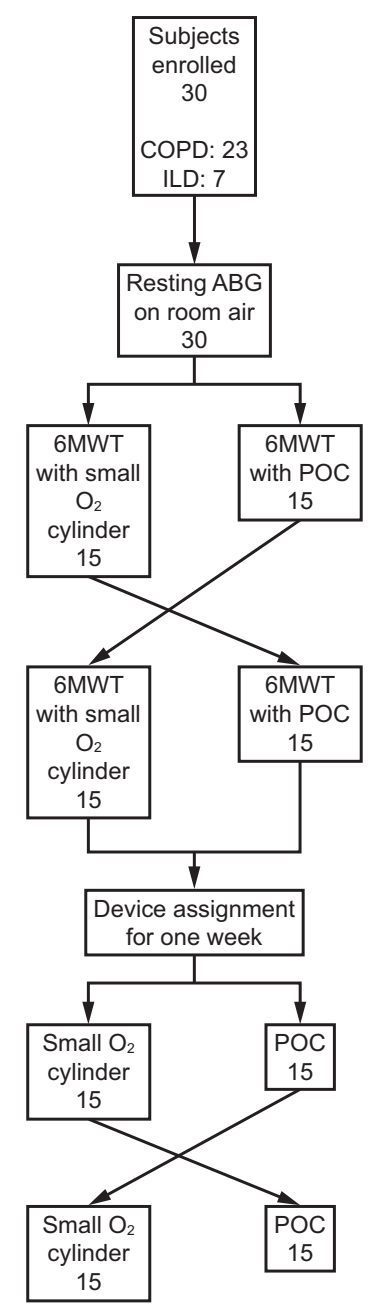

Fig. 1. Flow chart. ILD $=$ interstitial lung disease; $A B G=$ arterial blood gas; $6 \mathrm{MWT}=6$-min walk test; $\mathrm{POC}=$ portable oxygen concentrator.

the test using the modified Borg CR10 scale. ${ }^{14}$ The distance covered during the 6MWT was expressed in meters.

A 6MWT was performed while breathing ambient air at baseline to ascertain exercise desaturation. The subjects performed 2 further 6MWTs on 2 consecutive days, one using the small cylinder and the other using the POC. The settings for the small cylinder (expressed in $\mathrm{L} / \mathrm{min}$ ) and those for the POC (expressed as a numerical level between 1 and 5) are not comparable, so the devices were set so that subjects could maintain an oxygen saturation between $92 \%$ and $95 \%$ during the $6 \mathrm{MWT}$.

\section{Subject Preference}

Subject preference was assessed with a structured questionnaire that explored subjects' experiences with each device (Fig. 2). The questionnaire was administrated by a physician and included 5 questions related to several 


\section{Portable Concentrator vs Oxygen Cylinder}

Evaluation on the use of Oxygen Cylindercor Portable Oxygen Concentratora

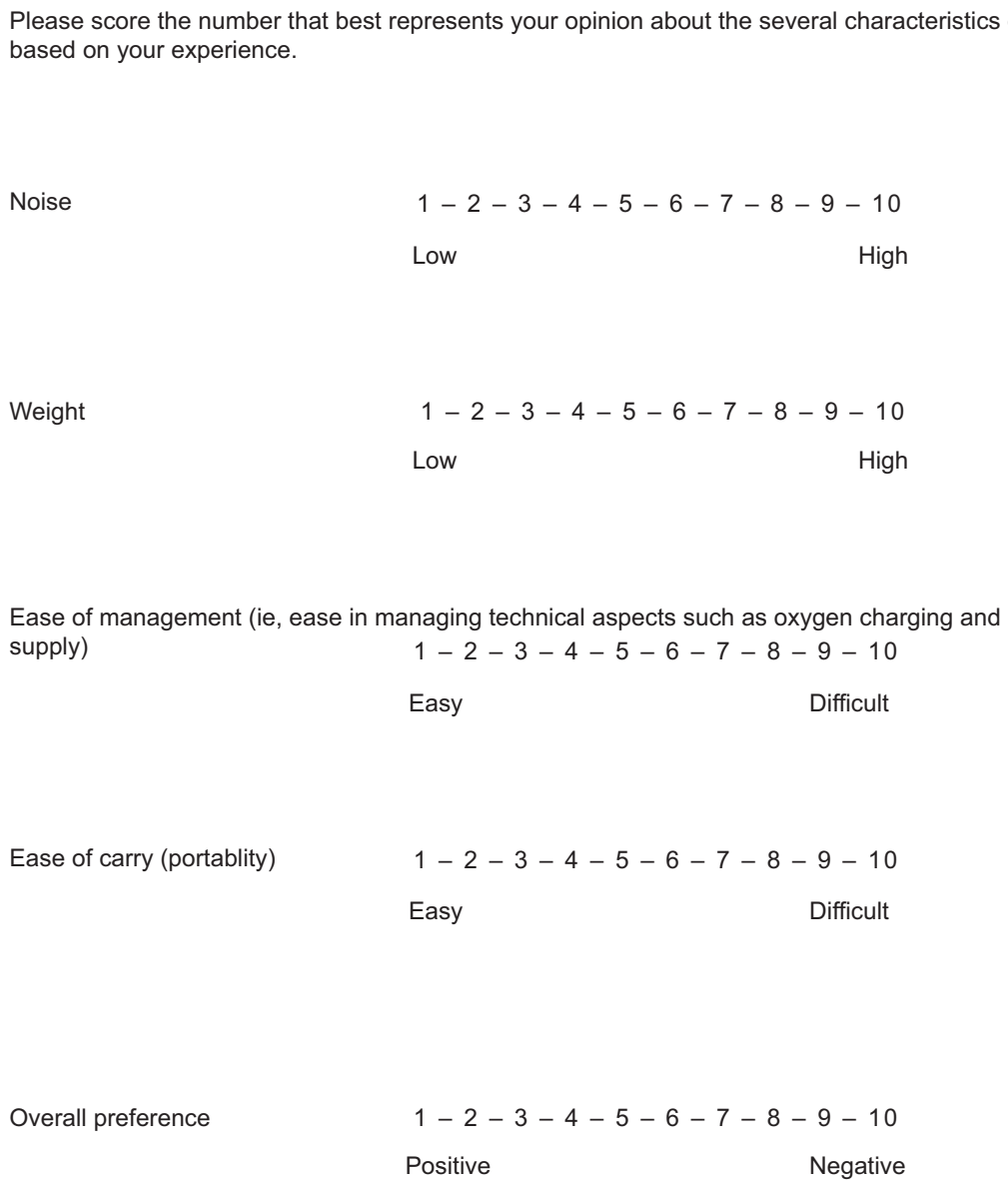

Fig. 2. Structured questionnaire that explored subjects' experience with each device.

aspects of the device: noise, weight, ease of management (ie, ease in managing technical aspects such as oxygen charging and supply), ease of carrying (ie, portability), and overall preference. Each question had a score ranging from 1 to 10 , with 1 indicating the best preference and 10 indicating the worst.

\section{Psychological Measures and QOL}

Anxiety and depression were assessed by means of the Hospital Anxiety and Depression Scale (HADS), which is widely used in rehabilitation settings ${ }^{15}$ and consists of 14 items, 7 of which are for the anxiety subscale (HADS Anxiety) and 7 of which are for the depression subscale (HADS Depression). Each item is scored on a response scale ranging between 0 and 3. After adjusting for 6 items that are reverse-scored, all responses are summed to obtain the 2 subscales. Scores ranging between 8 and 10 indicate doubtful cases, while scores $\geq 11$ indicate clinically relevant cases. ${ }^{16}$ A cutoff score $\geq 8$ can be considered as the optimal score for both sensitivity and specificity. ${ }^{17}$
QOL was assessed by means of the Satisfaction Profiles questionnaire (SAT-P). SAT-P is a reliable and valid tool to evaluate satisfaction profile of daily life, and it can be considered an indicator of subjective QOL. ${ }^{18}$ SAT-P quantitatively measures the cognitive product of the comparison between ideal life and reality. It consists of 32 items exploring several aspects of daily life. The subject is asked to evaluate, on a $10-\mathrm{cm}$, horizontal visual analog scale, satisfaction with reference to the last month, independent of the objective health status. The SAT-P provides 32 individual scores and 5 factor scores ranging from 0 (lowest level of satisfaction) to 100 (highest level of satisfaction). The 5 factors are psychological functionality; physical functionality; work functionality; sleep, nutrition, and hobbies; and social functionality.

\section{Statistical Analysis}

Continuous variables were reported as mean $\pm \mathrm{SD}$ or median (interquartile range). Categorical data were described as frequencies and percentage. Prevalence of anxiety and depression symptoms in the study sample were 


\section{Portable Concentrator vs Oxygen Cylinder}

calculated using standardized cutoff scores. Differences for the paired variables were tested with the Wilcoxon matched-pairs signed ranks test. Correlations were evaluated with the nonparametric cograduation Spearman's coefficient (rho). $P<.05$ was considered statistically significant. All analyses were conducted using STATA 14 (StataCorp, College Station, Texas).

\section{Results}

Thirty subjects (27 men and 3 female) were included in this study. Demographic and clinical characteristics of the subjects are shown in Table 1 . Mean age was $65 \pm 11 \mathrm{y}$. The compressed oxygen cylinder delivered oxygen with continuous flow set at $2.0 \pm 0.5 \mathrm{~L} / \mathrm{min}$, whereas the POC under test delivered a pulse flow on their setting of a mean of $3.0 \pm 0.5 \mathrm{~L} / \mathrm{min}$.

Table 1. Demographic and Clinical Characteristics of Subjects

\begin{tabular}{lc}
\hline \hline Subjects & 30 \\
Sex, female/male & $3 / 27$ \\
Age, y & $65 \pm 11$ \\
COPD & 23 \\
Interstitial lung disease & 7 \\
Body mass index, kg/m ${ }^{2}$ & $32 \pm 10$ \\
FEV $_{1}, \mathrm{~L}$ & $1.6 \pm 0.3$ \\
$\mathrm{FEV}_{1}, \%$ & $50 \pm 19$ \\
$\mathrm{FVC}, \mathrm{L}$ & $2.5 \pm 0.5$ \\
$\mathrm{FVC}^{\%} \%$ & $76 \pm 15$ \\
$\mathrm{FEV}_{1} / \mathrm{FVC}$ & $53 \pm 21$
\end{tabular}

Data are expressed as numbers or means $\pm \mathrm{SD}$.

Table 2. Measurements and Scores With Each of the Two Devices

\begin{tabular}{|c|c|c|c|c|}
\hline & Portable Oxygen Concentrator & Small Oxygen Cylinder & Difference* & $P$ \\
\hline 6MWD & $238(206-295)$ & $228(210-265)$ & $14(-20$ to 31$)$ & .12 \\
\hline Initial $\mathrm{S}_{\mathrm{pO}_{2}}, \%$ & 97 (97-98) & $97(96-98)$ & $0(-1$ to 1$)$ & .92 \\
\hline Final $\mathrm{S}_{\mathrm{pO}_{2}}, \%$ & $92(92-93)$ & $92(92-93)$ & $0(-1$ to 1$)$ & .88 \\
\hline Initial cardiac frequency, beats/min & $72(64-82)$ & $70(67-78)$ & $0(-2$ to 7$)$ & .35 \\
\hline Final cardiac frequency, beats/min & $98(89-103)$ & $101(94-107)$ & $0(-7$ to 7$)$ & .91 \\
\hline Initial breathing frequency, breaths/min & $16(14-17)$ & $15(14-17)$ & $0(-1$ to 1$)$ & .50 \\
\hline Final breathing frequency, breaths/min & $24(20-25)$ & $23(22-24)$ & $0(-2$ to 1$)$ & .23 \\
\hline Initial dyspnea & $1(1-2)$ & $2(1-3)$ & $-1(-2$ to 0$)$ & .07 \\
\hline Final dyspnea & $4(3-6)$ & $6(4-7)$ & $-1(-2$ to 0$)$ & .056 \\
\hline \multicolumn{5}{|l|}{ Subject preference } \\
\hline Ease of management & $2(1-2)$ & $2(1-4)$ & $-1(-2$ to 0$)$ & .02 \\
\hline Noisiness & $4(3-5)$ & $1(1-2)$ & $3(1$ to 4$)$ & $<.001$ \\
\hline Weight & $1(1-2)$ & $4(3-5)$ & $-3(-4$ to -2$)$ & $<.001$ \\
\hline Ease of carry & $1(1-2)$ & $4(3-5)$ & $-3(-4$ to -2$)$ & $<.001$ \\
\hline Overall preference & $2(1-2)$ & $4(3-5)$ & $-2(-3$ to -1$)$ & $<.001$ \\
\hline
\end{tabular}

\section{Functional Parameters}

Mean $\mathrm{S}_{\mathrm{pO}_{2}}$ values observed at baseline and during 6MWTs with oxygen supplied by the small cylinder and the POC under test were not statistically different $(P>$ $.05)$. Likewise, the distance covered during the 6MWT registered while using the small cylinder and the POC under test were not statistically significant $(P>.05)$. Borg scores for both dyspnea and fatigue post-walk between the devices were not significantly different. All comparisons are reported in Table 2. Three and four subjects had to stop and rest during 6MWT while using the POC under test and the small cylinder, respectively.

\section{Subject Preferences}

A higher preference for the SimplyGo Mini (ie, a lower score than with the small cylinder) was detected in 15 (50\%) subjects for the question about ease of management, 1 (3\%) subject for noisiness, $27(90 \%)$ subjects for weight and ease of carry, and 23 (76.7\%) subjects for the overall preference. With the exception of noisiness, a significantly better score was observed for the SimplyGo Mini than for the small cylinder in all questions and in overall preference (Table 2). The magnitude of preference for the SimplyGo Mini than the small cylinder (ie, the difference between scores) significantly correlated with ease of management and ease of carry (rho $=0.38, P=.041$ ), ease of management and overall preference (rho $=0.59, P=.001$ ), weight and ease of carry (rho $=0.72, P<.001)$, weight and overall preference $($ rho $=0.45, P=.01$ ), and ease of carry and overall preference (rho $=0.76, P<.001$ ). Among 
Portable Concentrator vs Oxygen Cylinder

Table 3. Correlations Between HADS Subscales and 5-Question Preferences Questionnaire

\begin{tabular}{|c|c|c|c|c|c|c|}
\hline \multirow{2}{*}{$\begin{array}{l}\text { Subject Preference } \\
\text { Questionnaire }\end{array}$} & \multicolumn{3}{|c|}{ Portable Oxygen Concentrator } & \multicolumn{3}{|c|}{ Small Oxygen Cylinder } \\
\hline & Age & HADS Anxiety & HADS Depression & Age & HADS Anxiety & HADS Depression \\
\hline Noise & 0.19 & 0.20 & 0.17 & 0.14 & 0.35 & 0.24 \\
\hline Weight & $0.55^{*}$ & 0.09 & 0.16 & $-0.57 *$ & 0.09 & 0.03 \\
\hline Ease of management & $0.48 *$ & 0.21 & 0.34 & $0.40^{*}$ & 0.34 & 0.11 \\
\hline Ease of carry & $0.50 *$ & 0.06 & 0.16 & $-0.49 *$ & 0.10 & 0.13 \\
\hline Overall preference & $0.51 *$ & 0.28 & 0.24 & $-0.46^{*}$ & 0.10 & 0.16 \\
\hline \multicolumn{7}{|c|}{$\begin{array}{l}\text { Data presented as Spearman's coefficients. } \\
\text { HADS = Hospital Anxiety and Depression Scale } \\
* P<.05 \text {. }\end{array}$} \\
\hline
\end{tabular}

demographic and clinical variables, a younger age showed a significant correlation with better preference for the SimplyGo Mini than for the small cylinder (difference between scores within patient) in terms of ease of management, weight, ease of carrying, and overall preference (Table 3).

Age was correlated with judgment about weight, ease to carry (portability), and overall preference for the small cylinder (Table 3). In particular, younger subjects judged more negatively the weight and the ease to carry (portability) of the small cylinder than older subjects did. On the contrary, age was correlated positively with ease of management scores for the POC under test (Spearman's rho $=0.40, P=$ .03). In particular, older subjects considered the POC under test easier to manage than younger subjects did. There were no significant differences in preferences between subjects with COPD and those with interstitial lung disease.

\section{Psychological Measures and QOL}

At baseline, 18 (60\%) subjects showed clinically relevant symptoms of anxiety (scores on HADS anxiety subscale greater than the cutoff value), and 13 (43\%) subjects had depression symptoms (scores on HADS depression subscale greater than the cutoff value). With respect to QOL measures, mean SAT-P subscale scores at study entry were $63 \%$ on psychological functionality; $34 \%$ on physical functionality; $57 \%$ on work functionality among 9 subjects who still worked; 56\% on sleep, nutrition, and hobbies; and 37\% on social functionality. The single-item analysis among SAT-P subscales showed lower scores on item 8 (mean score of $22 \%$ on physical mobility) and on item 18 (mean score of $23 \%$ on psychological autonomy).

The analysis of correlations between HADS subscales and SAT-P factor scores indicated that anxiety was correlated with the physical functionality subscale (rho $=0.58$, $P=.001$ ), whereas the depression subscale scores were correlated with both the physical functionality subscale of SAT-P (rho $=-0.82, P<.001)$ and the social functionality subscale of SAT-P (rho $=-0.58, P=.001)$. No association was found between HADS subscales and scores on the 5question preferences questionnaire (Table 3).

\section{Discussion}

To maximize the therapeutic benefits of ambulatory oxygen therapy, it is of paramount importance to ensure patient adherence in addition to adequate oxygenation. In this study, we explored the preferences of patients for 2 portable oxygen devices (ie, the small cylinder and the POC) in the rehabilitation setting. Subjects had respiratory impairment of composite etiologies, but all were characterized by exercise desaturation. A high proportion of subjects preferred the POC under test. Comparisons between scores on a questionnaire assessing preferences indicated that the POC under test was favored over the small cylinder in all of the examined aspects, with the exception of device noisiness.

In general, the POC under test was considered easier to manage (eg, recharging oxygen and assumption of oxygen), easier to transport, and lighter than the small cylinder. This preference did not depend upon the functional efficacy of oxygen delivery but was mainly based on a perceived better portability, possibly because, unlike the small cylinder, the POC is form fitting and designed for patient comfort. In fact, functional parameters about oxygen delivery clearly showed that the 2 devices delivered similar performance. Furthermore, the POC under test was perceived as lighter than the small cylinder, even though they are comparable in weight. The association between age and device preference represents the main result of our study. In particular, age seemed to influence the perception of the small cylinder weight. Older subjects considered the weight of the small cylinder more negatively than younger subjects. With regard to the perception of efficiency in oxygen supply, the 2 devices were considered similar.

Age also influenced the perception of ease of management. Older subjects tended to consider the management of the POC to be easier, while they considered the small cylinder to be heavier and more difficult to transport. These characteristics are likely considered to be of paramount importance because one of the main limitations that affects 


\section{Portable Concentrator vs Oxygen Cylinder}

QOL in patients with COPD is a dramatic reduction in mobility, as demonstrated by lower scores on physical mobility and social functionality subscales on the SAT-P. Moreover, these 2 features correlated with the presence of clinically relevant symptoms of anxiety and depression. With regard to these psychological symptoms, our sample showed a non-negligible presence of psychological distress and a reduced self-perceived QOL, which is in line with previous studies, mainly affecting mobility and psychological and functional independence. ${ }^{2,8}$ Independent of the level of psychological distress, subjects highly preferred the POC under test most likely because it was considered a promising tool to enhance mobility and QOL.

Guidelines recommend that patients needing LTOT who regularly go outside should be provided with a mobile oxygen-delivery device to improve their QOL. ${ }^{9}$ Indeed, LTOT supplied with ambulatory delivery devices increases the adherence of patients to treatment and allows them to participate more meaningfully in outdoor activities than those with stationary sources. ${ }^{19}$

Today, technological innovations have made different devices available to patients in need of LTOT. However, patient preference for a device, and whether it increases adherence or improves psychological condition, has received little attention. This aspect is of importance because, despite the positive effects of oxygen therapy, patient adherence to treatment remains an important problem. In a study involving subjects with COPD receiving LTOT, 59.2\% reported nonadherence to treatment due to difficulties in the use during physical activity and to social stigma and fear of side effects. ${ }^{20}$ The reasons behind patients' suboptimal adherence to LTOT include discomfort in being seen in public while receiving oxygen because it implies a smokingrelated illness, as well as a fear of addiction and local side effects such as nasal dryness, nasal bleeding, dizziness, etc.

To maximize the therapeutic benefits of ambulatory oxygen therapy, it is important to ensure patient adherence in addition to adequate oxygenation. POC is a valid alternative because it is free of constraints, has fewer safety risks, and offers more autonomy during travel; in addition, although the initial acquisition costs are high, these costs are quickly amortized over time. In this study, $48 \%$ of subjects declared their intent to use POC under test regularly in everyday life.

The small cylinder and the POC have different technical characteristics, such as flow (ie, continuous vs pulsed), the concentration of oxygen delivered (100\% vs $85-95 \%)$, among others, which must be considered before prescription. ${ }^{21,22}$ The dose settings on a POC, frequently labeled in numerals, do not correspond directly to flows delivered by the small cylinder, typically expressed in $\mathrm{L} / \mathrm{min}^{22}$ Furthermore, due to differences in respiratory dynamic response to exercise, a POC may be suitable for a patient with COPD but not for a patient with pulmonary fibrosis.
Some potential limitations of our study need to be discussed. First, the number of subjects was small and may not reflect the range of patients with COPD and interstitial lung disease. However, we included subjects with different types of interstitial lung disease and diverse levels of lung function impairment in COPD. Second, the case-mix was unbalanced with regard to gender because our sample was predominantly male. However, previous studies on comparisons between LTOT devices did not show significant effects of gender on subjects' preferences. ${ }^{10,12}$ Third, we used only one POC, and the findings from this study should not be generalized to other types of POCs, given that different POCs vary significantly in their technical specifications. More importantly, we evaluated only subjects with a low oxygen requirement, and adequate oxygenation during the 6MWT was achieved using either the POC under test or the oxygen cylinder. This may be not applicable to other patients where the available sources of ambulatory oxygen therapy may be inadequate to meet the increased oxygen demands during physical activity.

\section{Conclusions}

Patient preference for an oxygen therapy device is an important factor that can affect adherence and psychological status and encourage active involvement in outdoor social activities. However, the prescription of a specific device should be individualized and validated by an ambulatory walk test to establish whether the oxygen delivered is sufficient to correct the patient's desaturation during exercise and to determine effective settings. Future studies with larger samples and with a longitudinal approach may help clinicians understand the associations between variables involved in adherence to LTOT and oxygen-delivery devices and their effects on QOL and psychological distress.

\section{ACKNOWLEDGMENTS}

We thank Anna Ciullo for her valuable technical support.

\section{REFERENCES}

1. Du Plessis JP, Fernandes S, Jamal R, Camp P, Johannson K, Schaeffer $\mathrm{M}$, et al Exertional hypoxemia is more severe in fibrotic interstitial lung disease than in COPD. Respirology 2018;23(4):392-398.

2. Nonoyama ML, Brooks D, Guyatt GH, Goldstein RS. Effect of oxygen on health quality of life in patients with chronic obstructive pulmonary disease with transient exertional hypoxemia. Am J Respir Crit Care Med 2007;176(4):343-349.

3. Calverley PM. Supplementary oxygen therapy in COPD: is it really useful? Thorax 2000;55(7):537-538.

4. Continuous or nocturnal oxygen therapy in hypoxemic chronic obstructive lung disease: a clinical trial. Nocturnal Oxygen Therapy Trial Group. Ann Intern Med 1980;93(3):391-398.

5. Long term domiciliary oxygen therapy in chronic hypoxic cor pulmonale complicating chronic bronchitis and emphysema. Report of the 


\section{Portable Concentrator vs Oxygen Cylinder}

Medical Research Council Working Party. Lancet 1981;1(8222):681686.

6. Dheda K, Lim K, Ollivere B, Leftley J, Lampe FC, Salisbury A, et al Assessments for oxygen therapy in COPD: are we under correcting arterial oxygen tensions? Eur Respir J 2004;24(6):954-957.

7. Katsenos S, Constantopoulos SH. Long-term oxygen therapy in copd: factors affecting and ways of improving patient compliance. Pulm Med 2011;2011:325362.

8. Ring L, Danielson E. Patients' experiences of long-term oxygen therapy. J Adv Nurs 1997;26(2):337-344.

9. Melani AS, Sestini P, Rottoli P. Home oxygen therapy: re-thinking the role of devices. Expert Rev Clin Pharmacol 2018;11(3):279289.

10. AlMutairi HJ, Mussa CC, Lambert CT, Vines DL, Strickland SL. Perspectives from COPD subjects on portable long-term oxygen therapy devices. Respir Care 2018;63(11):1321-1330.

11. Schaanning J, Strom K, Boe J. Do patients using long-term liquid oxygen differ from those on traditional treatment with oxygen concentrators and/or compressed gas cylinders? A comparison of two national registers. Respir Med 1998;92(1):84-87.

12. Mussa CC, Tonyan L, Chen YF, Vines D. Perceived satisfaction with long-term oxygen delivery devices affects perceived mobility and quality of life of oxygen-dependent individuals with COPD. Respir Care 2018;63(1):11-19.

13. Holland AE, Spruit MA, Troosters T, Puhan MA, Pepin V, Saey D, et al An official European Respiratory Society/American Thoracic Society technical standard: field walking tests in chronic respiratory disease. Eur Respir J 2014;44(6):1428-1446.
14. Mahler DA, Horowitz MB. Perception of breathlessness during exercise in patients with respiratory disease. Med Sci Sports Exerc 1994; 26(9):1078-1081.

15. Pierobon A, Ranzini L, Torlaschi V, Sini Botelli E, Giardini A, Bruschi $\mathrm{C}$, et al Screening for neuropsychological impairment in COPD patients undergoing rehabilitation. PLoS One 2018;13(8):e0199736.

16. Snaith RP, Zigmond AS. The Hospital Anxiety and Depression Scale. Brit Med J 1986;292(6516):344-344.

17. Bjelland I, Dahl AA, Haug TT, Neckelmann D. The validity of the Hospital Anxiety and Depression Scale: an updated literature review. J Psychosom Res 2002;52(2):69-77.

18. Leone D, Moja EA, Vegni E. Satisfaction for quality of life: a comparison of patient and occupational therapist perspectives. Scand J Occup Ther 2013;20(4):315-320.

19. Lacasse Y, Lecours R, Pelletier C, Begin R, Maltais F. Randomized trial of ambulatory oxygen in oxygen-dependent COPD. Eur Respir J 2005;25(6): 1032-1038.

20. Verduri A, Ballerin L, Simoni M, Cellini M, Vagnoni E, Roversi P, et al. Poor adherence to guidelines for long-term oxygen therapy (LTOT) in two Italian university hospitals. Intern Emerg Med 2014;9 (3):319-324.

21. Chen JZ, Katz IM, Pichelin M, Zhu K, Caillibotte G, Finlay WH, Martin AR. In vitro-in silico comparison of pulsed oxygen delivery from portable oxygen concentrators versus continuous flow oxygen delivery. Respir Care 2019;64(2):117-129.

22. Chen JZ, Katz IM, Pichelin M, Zhu K, Caillibotte G, Noga ML, et al. Comparison of pulsed versus continuous oxygen delivery using realistic adult nasal airway replicas. Int J Chron Obstruct Pulmon Dis 2017;12:2559-2571.

This article is approved for Continuing Respiratory Care Education credit. For information and to obtain your CRCE

(free to AARC members) visit

www.rcjournal.com

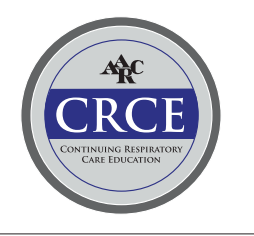

